\title{
Lipid profile disturbances may predispose psoriatic patients to liver dysfunction
}

\author{
Dorota Kozłowska ${ }^{1}$ Ewa Harasim-Symbor², Hanna Myśliwiec ${ }^{1}$, Anna J. Milewska ${ }^{3}$, Adrian Chabowski², Iwona Flisiak ${ }^{1}$
}

${ }^{1}$ Department of Dermatology and Venereology, Medical University of Bialystok, Bialystok, Poland

${ }^{2}$ Department of Physiology, Medical University of Bialystok, Bialystok, Poland

${ }^{3}$ Department of Statistics and Medical Informatics, Medical University of Bialystok, Bialystok, Poland

Adv Dermatol Allergol 2021; XXXVIII (2): 310-318

DOI: https://doi.org/10.5114/ada.2021.106209

\begin{abstract}
Introduction: Psoriasis is a chronic inflammatory disease associated with metabolic disturbances and liver dysfunction. Both serum fatty acids (FA) and ceramides (Cer) have structural functions but also are signal molecules that could be involved in the pathogenesis of liver dysfunction.

Aim: To assess the concentration of the circulating FA and Cer in correlation with the alanine aminotransferase (ALT) blood level in psoriatic patients. In addition, we have examined the relationship between ALT concentration and severity of the disease and inflammation markers.

Material and methods: Eighty-five patients with psoriasis and 32 healthy controls were enrolled in the study. Patients were divided into 2 groups according to ALT blood levels. Serum concentration of 14 FA and 14 Cer were measured by gas-liquid chromatography. The results were correlated with the Psoriasis Area and Severity Index (PASI), serum lipid profile, and inflammatory markers.

Results: We observed higher PASI score $(p=0.01)$ and higher C-reactive protein $(p=0.02)$ concentration in the group of psoriatic patients with high ALT. Serum ALT positively correlated with saturated fatty acids (SFA) $(p=0.01, r=0.27)$ and SFA/unsaturated fatty acids (UFA) ratio $(p=0.01, r=0.26)$. ALT negatively correlated with UFA level $(p=0.008$, $r=-0.28)$. Lignoceric ceramide positively correlated with ALT level $(r=0.22 ; p=0.045)$ in psoriatic patients.

Conclusions: Patients with severe psoriasis are predisposed to the development of liver dysfunction. We have demonstrated disturbances of serum fatty acid and sphingolipid profile in psoriatic patients, which may trigger liver disease.
\end{abstract}

Key words: psoriasis, ceramide, fatty acid, liver disease.

\section{Introduction}

Psoriasis is a chronic, inflammatory skin disease associated with many cardiometabolic comorbidities, which are components of a metabolic syndrome (MetS). Its prevalence is $2-3 \%$ of the population worldwide $[1,2]$. Psoriasis is closely related to components of metabolic syndrome, such as obesity, dyslipidaemia, type 2 diabetes, and arterial hypertension [3]. For this reason, psoriatic patients are characterized by increased mortality due to cardiovascular complications compared to healthy people. It is believed that chronic, low-grade inflammatory status with increased production of pro-inflammatory cytokines is the background responsible for metabolic dysregulation and higher cardiovascular disease (CVD) risk in this skin disorder [3-5].

Nonalcoholic fatty liver disease (NAFLD) is the most common cause of chronic liver disease in Western countries, and its comorbidity is closely associated with obesity. Recently NAFLD has been predominantly regarded as a hepatic manifestation of metabolic syndrome, which can lead to many severe liver complications, such as fibrosis, cirrhosis, and even hepatic cellular carcinoma [6-8].

Many recent studies have confirmed the association between psoriasis and NAFLD. Both disorders are closely related to metabolic syndrome, and their background is associated with chronic inflammatory status $[9,10]$. Although the pathogenesis of both NAFLD and psoriasis

Address for correspondence: Dorota Kozłowska, Department of Dermatology and Venereology, Medical University of Bialystok, 14 Żurawia St, 15-540 Białystok, Poland, phone: +48 85 7409572, fax: +48 85 7409406, e-mail: dorota.kozlowska@umb.edu.pl Received: 19.12.2018, accepted: 28.11.2019. 
seems to be multifactorial, the exact connection between these 2 diseases has not been fully explained. Recent studies have shown a higher prevalence of NAFLD among psoriatic patients than in the overall population, and its morbidity may affect as much as 50\% of all psoriatic cases $[11,12]$. Furthermore, psoriatic patients more often develop advanced forms of NAFLD than healthy people $[13,14]$. In the case of psoriatic arthritis development the risk may be even higher [15]. Patients with confirmed NAFLD have more severe course of skin lesions in comparison with psoriatic patients without comorbidities [9]. In addition, it has been demonstrated that psoriatic patients with diagnosed NAFLD are more likely to have MetS than those with psoriasis alone. This group also tended to have higher frequency of abnormal liver function test (LFT) results and higher levels of serum lipids (cholesterol, TAG, and LDL) [15].

Histological features of NAFLD consist of a wide spectrum of liver conditions ranging from mild steatosis, through nonalcoholic steatohepatosis, to advanced fibrosis and cirrhosis. Lipid accumulation in the liver, in particular free fatty acids (FFA), leads to lipotoxicity and consequently to mitochondrial dysfunction, oxidative stress, and activation of inflammatory pathways, which represent further liver damage $[9,11,16]$. Circulating ceramides in serum, the level of which is decreased in psoriasis, also take part in the development of metabolic syndrome. They interact with several pathways involved in oxidative stress, inflammation, apoptosis, and insulin resistance, which are closely linked to NAFLD $[17,18]$.

We have observed in our recent studies numerous abnormalities of circulating fatty acids and sphingolipids in psoriatic patients $[19,20]$. These parameters might be a link between psoriasis and possible development of NAFLD in psoriatic patients, or at least they might act as an indicators of liver damage assessed by elevating liver enzymes.

\section{Aim}

The aim of our study was to assess the concentration of the circulating fatty acids and ceramides in correlation with the alanine aminotransferase (ALT) blood level in psoriatic patients. In addition, we have examined the relationship between ALT concentration and the severity of the disease and inflammation markers.

\section{Material and methods}

Eighty-five individuals with plaque-type psoriasis and 32 sex- and age-matched healthy controls participated in the study; 28 of them were women and 57 were men. The median age of patients was 53 (from 19 to 79 years). The severity of psoriasis was estimated using the Psoriasis Area and Severity Index (PASI).

Body mass index (BMI) was evaluated as weight/ height $^{2}\left(\mathrm{~kg} / \mathrm{m}^{2}\right)$. Overweight was defined as BMI $\geq 25 \mathrm{~kg} /$ $\mathrm{m}^{2}$ and obesity as BMI $\geq 30 \mathrm{~kg} / \mathrm{m}^{2}$. None of the patients and controls had any dietary restrictions. Alcohol addiction was an exclusion criterion. All patients enrolled in the study were treated with topical ointment (dithranol, vaselin with salicylic acid).

The history of comorbidities (such as type 2 diabetes, arterial hypertension, weight abnormalities) as well as the results of the laboratory tests were collected from hospital records of the patients hospitalized at the Dermatology and Venereology Department of the Medical University. All biochemical analyses, including morphology, C-reactive protein (CRP), serum fasting blood glucose (FBG), total cholesterol, triacylglycerols (TAG), and ALT, were carried out in the Central Laboratory of the University Hospital Centre. According to the laboratory, the correct level of ALT concentration is in the range $0-41 \mathrm{IU} / \mathrm{l}$. All patients and controls gave their written informed consent before the enrolment. The study was approved by the local bioethical committee (R-I-002/457/2016).

Peripheral blood samples were taken before starting the treatment from patients and from the control group. After centrifugation, the serum was stored at $-80^{\circ} \mathrm{C}$ until analyses.

Total serum fatty acid content and composition was measured according to the method of Glaser et al. [21]. Briefly, $100 \mu \mathrm{l}$ of serum was incubated at $85^{\circ} \mathrm{C}$ for $45 \mathrm{~min}$ in $1.5 \mathrm{ml}$ of $3 \mathrm{~N}$ methanolic $\mathrm{HCl}$ containing $2 \mathrm{~g} / \mathrm{l}$ BHT (2,6-di-tert-butyl-p-cresol, antioxidant). Prior to incubation, $100 \mu$ l of internal standard mixture (heptadecanoic acid/cholesteryl-heptadecanoate/triheptadecanoate/ diheptadecanoate/diheptadecanoyl-phosphatidylcholine; 0.2/2/1.5/0.2/2 per weight, $10 \mu \mathrm{g}$ of C17 : 0 total, in chloroform/methanol solution; $2: 1$, by vol) was added to account for methylation and extraction losses. After cooling to room temperature, fatty acids methyl esters were extracted with $0.5 \mathrm{ml}$ hexane, followed by 30 s of vortexing and centrifugation ( $5 \mathrm{~min} / 3000 \mathrm{~g}$ ). The volume of the upper organic phase was transferred into new glass vials then dried under nitrogen flow, and lipids were dissolved in $50 \mu \mathrm{l}$ of hexane. Thereafter, samples were analysed by gas-liquid chromatography using a Hewlett-Packard 5890 Series II gas chromatograph, an Agilent J\&W CP-Sil 88 capillary column (50 $\mathrm{m} \times 0.25 \mathrm{~mm}$ ID), and a flame-ionization detector. The oven temperature was programmed from $130^{\circ} \mathrm{C}$ to $220^{\circ} \mathrm{C}$ at $5^{\circ} \mathrm{C} / \mathrm{min}$ and held at $220^{\circ} \mathrm{C}$ for $32 \mathrm{~min}$. Argon was used as a carrier gas. The following fatty acid species were identified and quantified according to the respective retention times of synthetic standards: myristic $(14: 0)$, palmitic $(16: 0)$, palmitoleic $(16: 1 n-7)$, stearic $(18: 0)$, oleic $(18: 1 n-9)$, linoleic $(18: 2 n-6)$, $\alpha$-linolenic (18:3n-3), arachidic (20:0), arachidonic (20:4n$6)$, eicosapentaenoic (20:5n-3), behenic (22:0), docosahexaenoic (22:6n-3), lignoceric (24:0), and nervonic (24:1n-9) acids. The FA were additionally grouped according to their biologic properties. The percentage of saturated FA-SFA (myristic acid, palmitic acid, stearic acid, arachidic acid, behenic acid, lignoceric acid) was measured and calculated. Unsaturated fatty acids (UFA) were di- 
vided into monounsaturated FA-MUFA (palmitoleic acid, oleic acid, nervonic acid) and polyunsaturated FA-PUFA ( $n$-3 PUFA: $\alpha$-linolenic acid, eicosapentaenoic acid (EPA), docosahexaenoic acid (DHA), and n-6 PUFA: linoleic acid, arachidonic acid).

To measure sphingosine-1-phosphate (S1P), the serum samples were mixed with a solution composed of $25 \mathrm{mM} \mathrm{HCl}$ and $1 \mathrm{M} \mathrm{NaCl}$ and acidified with methanol. Internal standards of C17-sphingosine and C17-sphingosine 1-phosphate were added. Lipids were extracted by means of chloroform, $1 \mathrm{M} \mathrm{NaCl}$ and $3 \mathrm{~N} \mathrm{NaOH}$. The aqueous phase containing S1P was transferred to a fresh tube and the compound was dephosphorylated with the use of alkaline phosphatase (bovine intestinal mucosa). Free sphingosine was converted to its O-phthalaldehyde derivatives and analysed by means of a high-performance liquid chromatography (HPLC) system equipped with a fluorescence detector and $\mathrm{C} 18$ reversed-phase column (Varian Inc., OmniSpher 5, $4.6 \times 150 \mathrm{~mm}$ ).

To quantify ceramides (Cer), a small volume of the chloroform phase containing lipids was transferred to a tube containing N-palmitoyl-D-erythro-sphingosine (C17 base) as an internal standard. The lipid fractions were separated by thin-layer chromatography silica plates (Kieselgel 60, $0.22 \mathrm{~mm}$, Merck, Darmstadt, Germany) with a heptane/isopropyl ether/acetic acid (60:40:3, by vol) resolving solution. Lipid bands were visualized by spraying with a $0.2 \%$ solution of 3'7'-dichlorofluorescin in methanol and identified under ultraviolet light using standards on the plates. The gel bands were scraped off the plate, transferred into screw tubes, and transmethylated with BF3/methanol. The fatty acid methyl esters were dissolved in hexane and analysed by gas-liquid chromatography. A Hewlett-Packard 5890 Series II gas chromatograph with Varian CP-SIL capillary column (50 m × $0.25 \mathrm{~mm} \mathrm{ID})$ and flame-ionization detector
(Agilent Technologies, Santa Clara, CA) was used. Injector and detector temperatures were set at $250^{\circ} \mathrm{C}$. The oven temperature was increased linearly from $160^{\circ} \mathrm{C}$ to $225^{\circ} \mathrm{C}$ at a rate of $5^{\circ} \mathrm{C} / \mathrm{min}$. According to the retention times of standards, the individual long-chain fatty acids were quantified. Total content of Cer was estimated as the sum of the particular fatty acid species of the assessed fraction, and it was expressed in nanomoles per millilitre of the serum.

\section{Statistical analysis}

The data analyses were carried out using Statistica 12.0 software. We examined the distribution of each continuous variable using the Shapiro-Wilk test. We did not find a normal distribution of the analysed variables. Data are presented as median and quartiles (first and third quartile) and percentage when appropriate. The statistical analyses were performed using the Kruskal-Wallis and Mann-Whitney tests. We calculated the Spearman rank correlation coefficients to measure the relationships. Results on the level $p<0.05$ were regarded as significant.

\section{Results}

Eighty-five patients ( 27 females and 57 males) with exacerbated plaque-type psoriasis and 32 healthy controls were enrolled in the study. The age of patients ranged from 19 to 79 years (mean: $49.7 \pm 14.4$ years). The mean duration of the disease was $18.5 \pm 14.4$ months (from 1 to 58 months). In the examined group 58.8\% of patients $(n=50)$ had mild psoriasis, $25.8 \%$ of them $(n=22)$ had moderate psoriasis, and $15.3 \%(n=13)$ of all studied patients were diagnosed as having a severe form of the disease. Among studied groups we observed lipid abnormalities: 16 (18.8\%) patients had hypercholesterolaemia (> $200 \mathrm{mg} / \mathrm{dl}$ ) and 15 (17.6\%) had hypertriglyc-

Table 1. Clinical and laboratory characteristics of the psoriatic patients with ALT serum concentration within normal range and with ALT above the limit. Data are shown as median and quartiles (Q1 first quartile; Q3 third quartile). Significant differences between the groups are shown as: ${ }^{*} p<0.05$

\begin{tabular}{lccc}
\hline Characteristics & $\begin{array}{c}\text { Psoriasis } \\
\text { normal ALT } \\
(n=73)\end{array}$ & $\begin{array}{c}\text { Psoriasis } \\
\text { ALT above the limit } \\
(n=12)\end{array}$ & $P$-value \\
\hline Age [years] & $54.0(41.0-59.0)$ & $47.5(41.5-50.5)$ & NS \\
\hline Psoriasis duration [months] & $17.0(6.0-28.0)$ & $20.0(6.5-29.5)$ & NS \\
\hline PASI & $8.0(5.0-12.5)$ & $14.9(10.6-22.0)$ & $0.011^{*}$ \\
\hline BMl & $27.2(23.9-30.9)$ & $28.8(24.5-37.2)$ & NS \\
\hline CRP $[\mathrm{mg} / \mathrm{dl}]$ & $2.4(1.0-4.9)$ & $6.5(2.9-10.6)$ & $0.025^{*}$ \\
\hline WBC $\left[\times 10^{3} / \mathrm{ml}\right]$ & $6.9(5.8-8.2)$ & $7.3(6.4-7.7)$ & NS \\
\hline Glucose $[\mathrm{mg} / \mathrm{dl}]$ & $87.0(77.0-98.0)$ & $94.0(84.0-101.0)$ & NS \\
\hline Vitamin D $[\mathrm{ng} / \mathrm{ml}]$ & $15.3(11.6-22.4)$ & $13.9(6.7-21.2)$ & NS \\
\hline Cholesterol $[\mathrm{mg} / \mathrm{dl}]$ & $175.0(153.0-199.0)$ & $185.0(162.0-194.0)$ & NS \\
\hline Triglyceride $[\mathrm{mg} / \mathrm{dl}]$ & $109.0(79.0-149.0)$ & $105.0(77.0-151.0)$ & NS \\
\hline
\end{tabular}

PASI - psoriasis area and severity index, BMI - body mass index, CRP - C-reactive protein, WBC - white blood cells, NS - non significant. 
eridaemia (> $160 \mathrm{mg} / \mathrm{dl}$ ). Selected demographic, clinical, and laboratory patient data are presented in Table 1.

Patients were divided into 2 groups according to the blood levels of ALT. The median value of ALT in the examined group of patients was 22 (minimum value was 7.0; maximum value was 94.0). In 73 cases the serum level of ALT was within the normal range, while in 12 of them ALT was above the reference limit.

We observed higher PASI score $(p=0.01)$ and higher CRP $(p=0.02)$ concentration in the group of patients with ALT above the normal range, compared to patients with ALT within the normal range (Figure 1). We observed that the level of ALT was higher in psoriatic patients with obesity $\left(\mathrm{BMI} \geq 30 \mathrm{~kg} / \mathrm{m}^{2}\right)(n=27,31.7 \%)$ in comparison to patients with normal body weight $(p=0.02)$. Additionally, the serum ALT level positively correlated with body weight and $\mathrm{BMI}$ in all psoriatic individuals ( $p=0.004$, $r=0.31$ and $p=0.027, r=0.24$, respectively). We did not observe significant correlations between ALT and PASI, blood glucose, and inflammatory markers (CRP, white blood cell count), nor cholesterol and triglyceride concentration. The correlations of ALT with FA and Cer are shown in Table 2.

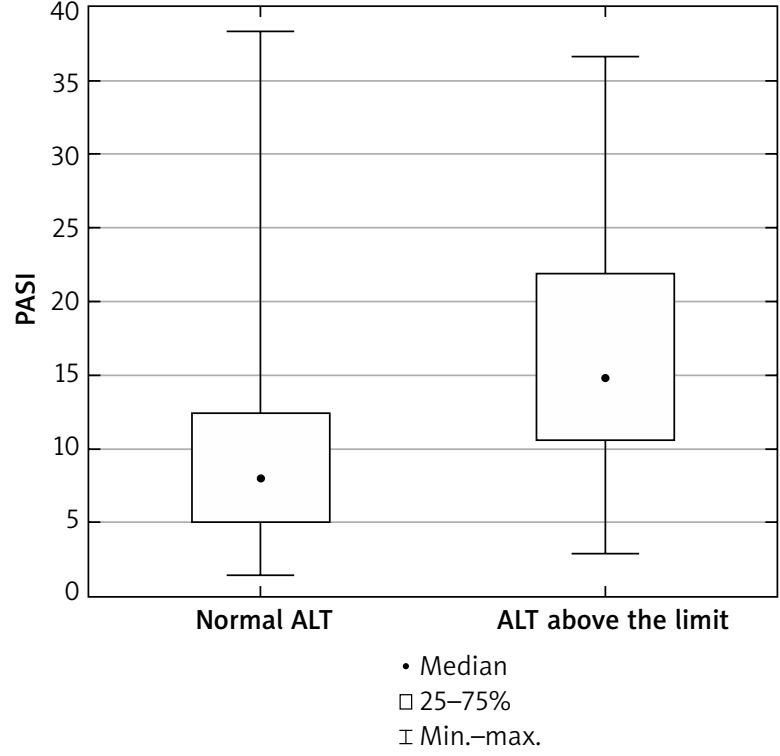

Figure 1. Severity of the disease (PASI) in psoriatic patients with ALT serum concentration within normal range and with ALT above the limit. Data shown as median (Q1, Q 3). Significant difference between the groups $p=0.011^{*}$

Table 2. Correlation between serum ALT and FA and Cer in psoriatic patients $(n=85)$. Significant correlations are marked as: ${ }^{*} p<0.05$

\begin{tabular}{|c|c|c|c|c|c|}
\hline Fatty acid & $r$ & $P$-value & Ceramide & $r$ & $P$-value \\
\hline Myristic (14:0) & 0.17 & 0.116 & Cer myristic (C14:0) & -0.02 & 0.847 \\
\hline Palmitic (16:0) & 0.16 & 0.147 & Cer. palmitic (C16:0) & 0.03 & 0.768 \\
\hline Palmitoleic (16:1n-7) & 0.15 & 0.173 & Cer palmitoleic (C16:1) & 0.19 & 0.086 \\
\hline Stearic (18:0) & 0.18 & 0.102 & Cer stearic (C18:0) & 0.05 & 0.659 \\
\hline Oleic (18:1n9c) & 0.12 & 0.270 & Cer oleic (C18:1n9) & 0.17 & 0.117 \\
\hline Linoleic $(18: 2 n-6)$ & -0.07 & 0.516 & Cer linoleic (C18:2) & -0.03 & 0.788 \\
\hline Arachidic (20:0) & -0.04 & 0.748 & Cer arachidic (C20:0) & -0.02 & 0.865 \\
\hline$\alpha$-Linolenic (18:3n-3) & 0.01 & 0.919 & Cer $\alpha$-Linolenic (C18:3) & -0.04 & 0.738 \\
\hline Behenic (22:0) & -0.14 & 0.206 & Cer behenic (C22:0) & 0.20 & 0.065 \\
\hline Arachidonic $(20: 4 n-6)$ & -0.00 & 0.993 & Cer arachidonic (C20:4) & -0.03 & 0.813 \\
\hline Lignoceric (24:0) & -0.11 & 0.329 & Cer lignoceric (C24:0) & 0.22 & $0.045^{*}$ \\
\hline Eicosapentaenoic (20:5n-3) & 0.01 & 0.923 & Cer eicosapentaenoic (C20:5) & 0.13 & 0.231 \\
\hline Nervonic (24:1n-9) & -0.13 & 0.235 & Cer nervonic (C24:1) & 0.06 & 0.613 \\
\hline Docosahexaenoic (22:6n-3) & -0.07 & 0.549 & Cer docosahexaenoic (C22:6) & 0.20 & 0.069 \\
\hline Total FA & 0.12 & 0.270 & Total Cer & 0.12 & 0.280 \\
\hline n-3 PUFA & -0.04 & 0.693 & Sphingosine-1-phosphate & -0.02 & 0.877 \\
\hline n-6 PUFA & -0.08 & 0.477 & & & \\
\hline$n-6 / n-3$ ratio & 0.00 & 0.994 & & & \\
\hline$\%$ SFA & 0.27 & $0.013^{*}$ & & & \\
\hline$\%$ MUFA & 0.10 & 0.346 & & & \\
\hline \%PUFA & -0.19 & 0.078 & & & \\
\hline SFA/UFA & 0.27 & $0.014^{*}$ & & & \\
\hline
\end{tabular}


The mean total FA serum concentration in all psoriatic patients was $3642.2 \pm 859.3 \mathrm{mg} / \mathrm{l}$, and in the control group $3700.4 \pm 671.3 \mathrm{mg} / \mathrm{l}$. Both psoriatic groups of patients had abnormal serum profile of FA compared to the healthy controls. We did not find any significant differences in particular FAs between the 2 psoriatic subgroups with elevated and normal ALT (Table 3). In all examined psoriatic patients the serum concentration of SFA correlated positively with the ALT level in serum $(p=0.01, r=0.27)$, while we observed negative correla- tion between concentration of UFA and ALT level ( $p=$ $0.008, r=-0.28)$. Furthermore, we found a positive correlation between the SFA/UFA ratio and the ALT level $(p=0.01, r=0.26)$ (Figure 2).

The total serum Cer concentration in psoriatic patients was $28.49 \pm 6.84 \mathrm{nmol} / \mathrm{l}$, which did not correlate with the ALT level in serum. In the healthy control group the total serum concentration of Cer was $31.1 \pm 5.8 \mathrm{nmol} / \mathrm{l}$. The serum concentrations of myristic ceramide (C14:0) and sphingosine-1-phosphate were higher ( $p=0.024$ and $p=0.025$, respectively) in

Table 3. Differences between serum FA concentrations [ $\mathrm{mg} / \mathrm{l}]$ in psoriatic patients with ALT serum concentration within normal range and with ALT above the limit, and the control group. Data are shown as median and quartiles (Q1 first quartile, Q3 third quartile). Significant differences between the psoriatic patients and the control group are shown as: ${ }^{*} p<0.05 ;{ }^{* *} p<0.01 ;{ }^{* * *} p<0.001$

\begin{tabular}{|c|c|c|c|}
\hline Fatty acid & $\begin{array}{l}\text { Psoriasis } \\
\text { normal ALT } \\
\quad(n=73)\end{array}$ & $\begin{array}{l}\text { Psoriasis } \\
\text { ALT above the limit } \\
(n=12)\end{array}$ & $\begin{array}{l}\text { Control } \\
(n=32)\end{array}$ \\
\hline Myristic (14:0) & $25.8(20.4-40.3)$ & $28.7(26.4-37.1)$ & $34.8(23.5-56.5)$ \\
\hline Palmitic (16:0) & $802.2(716.1-991.6)$ & 871.3 (743.9-1018.2) & 755.9 (707.9-959.2) \\
\hline Palmitoleic (16:1n-7) & $\begin{array}{c}100.6(74.0-154.0)^{* *} \\
p=0.009\end{array}$ & $\begin{array}{c}124.5(95.4-140.9)^{*} \\
p=0.013\end{array}$ & $75.7(55.4-101.6)$ \\
\hline Stearic (18:0) & 234.5 (200.7-271.8) & $253.4(224.0-303.8)$ & $250.5(233.2-278.4)$ \\
\hline Oleic (18:1n9c) & $827.4(741.6-1045.5)$ & $906.8(776.1-1140.8)$ & $787.1(702.8-881.4)$ \\
\hline Linoleic $(18: 2 n-6)$ & $\begin{array}{c}971.9(840.5-1091.9)^{\star \star \star} \\
p<0.001\end{array}$ & $\begin{array}{c}956.4(915.2-1028.2)^{\star} \\
p=0.033\end{array}$ & $1102.5(1013.5-1229.9)$ \\
\hline Arachidic (20:0) & $\begin{array}{c}7.6(6.5-8.8)^{\star \star \star} \\
p<0.001\end{array}$ & $\begin{array}{c}7.5(6.4-9.0)^{\star \star} \\
p=0.002\end{array}$ & $9.9(8.1-11.5)$ \\
\hline$\alpha$-Linolenic (18:3n-3) & $\begin{array}{c}20.5(16.0-27.2)^{\star * *} \\
p<0.001\end{array}$ & $\begin{array}{c}21.3(19.3-24.1)^{\star} \\
p=0.043\end{array}$ & $29.8(23.1-37.1)$ \\
\hline Behenic (22:0) & $\begin{array}{c}15.4(13.4-18.0)^{\star \star *} \\
p<0.001\end{array}$ & $\begin{array}{c}14.5(13.7-17.7)^{\star \star} \\
p=0.005\end{array}$ & $19.5(16.9-22.5)$ \\
\hline Arachidonic $(20: 4 n-6)$ & $253.6(216.1-299.6)$ & $253.2(216.4-282.8)$ & $285.7(243.5-315.2)$ \\
\hline Lignoceric (24:0) & $\begin{array}{c}10.7(9.2-12.3)^{* * *} \\
p<0.001\end{array}$ & $\begin{array}{c}10.3(7.9-13.2)^{* * *} \\
p=0.001\end{array}$ & $14.2(12.1-17.0)$ \\
\hline Eicosapentaenoic (20:5n-3) & $\begin{array}{c}32.0(22.8-45.9)^{\star *} \\
\quad p=0.002\end{array}$ & $33.6(21.6-51.6)$ & $48.0(36.4-63.4)$ \\
\hline Nervonic (24:1n-9) & $42.6(36.7-48.8)$ & $43.5(40.9-51.1)$ & $45.0(41.6-50.8)$ \\
\hline Docosahexaenoic (22:6n-3) & $70.5(54.3-87.8)$ & $63.8(47.2-92.4)$ & $80.8(67.6-89.9)$ \\
\hline Total FA & $3455.8(3093-4021.3)$ & 3659.7 (3267.9-4141.1) & $3556.1(3250.8-4147.2)$ \\
\hline$n-3$ PUFA & $\begin{array}{c}125.4(96.5-156.6)^{\star \star} \\
p=0.003\end{array}$ & $111.9(107.9-158.8)$ & $158.7(135.7-185.7)$ \\
\hline n-6 PUFA & $\begin{array}{c}1244.5(1065.1-1371.1)^{\star \star *} \\
p<0.001\end{array}$ & $\begin{array}{c}1231.0(1153.6-1294.3)^{*} \\
p=0.016\end{array}$ & $1411.3(1264.9-1529.1)$ \\
\hline$n-6 / n-3$ ratio & $10.1(7.9-11.8)$ & $10.4(8.2-11.7)$ & $9.1(7.2-10.4)$ \\
\hline$\%$ SFA & $32.1(31.4-33.1)$ & $32.7(31.7-34.6)$ & $31.3(30.0-32.8)$ \\
\hline$\%$ MUFA & $\begin{array}{c}29.0(26.7-31.8)^{\star * *} \\
p<0.001\end{array}$ & $\begin{array}{c}29.8(28.7-31.9)^{\star * *} \\
p<0.001\end{array}$ & $25.5(23.4-27.1)$ \\
\hline \%PUFA & $\begin{array}{c}38.8(35.2-41.6)^{* * *} \\
p<0.001\end{array}$ & $\begin{array}{c}37.8(33.6-39.6)^{\star \star *} \\
p<0.001\end{array}$ & $43.2(40.6-45.7)$ \\
\hline SFA/UFA & $0.47(0.46-0.49)$ & $0.48(0.46-0.53)$ & $0.45(0.43-0.49)$ \\
\hline
\end{tabular}


patients with high serum ALT than in healthy controls. All differences between serum Cer concentrations in the 2 groups of patients and controls are shown in Table 4. In the whole examined psoriatic group the serum concentration of lignoceric ceramide (C24:0) correlated positively with the ALT level $(r=0.22 ; p=0.045)$.

\section{Discussion}

To our knowledge, the association of circulating FA and Cer with liver dysfunction in psoriatic patients had not yet been studied. In our study we observed higher PASI score and CRP concentration in patients with ALT above the normal range. This confirms previous study results indicating the important role of the severity of the disease in the development of liver dysfunction $[9,13]$. The serum ALT level correlated positively with body weight and $\mathrm{BMI}$, which seems to confirm that NAFLD may be the hepatic manifestation of obesity and MetS also in psoriatic patients [8].

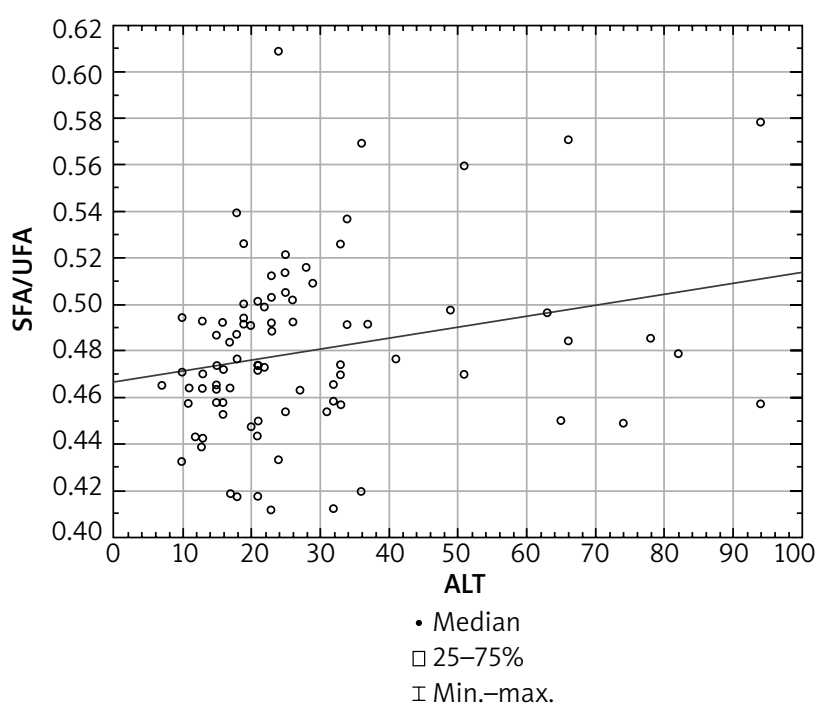

Figure 2. Correlation of ALT with SFA/UFA index in psoriatic patients $(n=85)(r=0.27, p=0.014)$

Table 4. Differences between serum Cer concentrations $[\mathrm{nmol} / \mathrm{ml}]$ and sphingosine-1 phosphate $[\mathrm{pmol} / \mathrm{ml}]$ in psoriatic patients with ALT serum concentration within normal range and with ALT above the limit and control group. Data are shown as median and quartiles (Q1 first quartile, Q3 third quartile). Significant differences between the psoriatic patients and the control group are shown as: ${ }^{*} p<0.05 ;{ }^{* *} p<0.01 ;{ }^{* * *} p<0.001$

\begin{tabular}{|c|c|c|c|}
\hline Ceramide & $\begin{array}{l}\text { Psoriasis } \\
\text { normal ALT } \\
(n=73)\end{array}$ & $\begin{array}{c}\text { Psoriasis } \\
\text { ALT above the limit } \\
(n=12)\end{array}$ & $\begin{array}{l}\text { Control } \\
(n=32)\end{array}$ \\
\hline Cer myristic (C14:0) & $\begin{array}{c}1.4(1.1-3.9)^{*} \\
p=0.015\end{array}$ & $\begin{array}{c}1.4(0.9-1.9)^{*} \\
p=0.024\end{array}$ & $3.6(1.2-5.0)$ \\
\hline Cer. palmitic (C16:0) & $8.0(6.5-9.6)$ & $8.3(6.2-10.8)$ & $7.9(7.0-9.1)$ \\
\hline Cer palmitoleic (C16:1) & $0.7(0.6-1.1)$ & $0.7(0.5-0.9)$ & $0.9(0.7-1.1)$ \\
\hline Cer stearic (C18:0) & $6.6(5.5-7.7)$ & $6.9(5.6-8.5)$ & $5.6(4.7-7.5)$ \\
\hline Cer oleic (C18:1n9) & $\begin{array}{c}1.8(1.7-2.1)^{\star *} \\
p=0.005\end{array}$ & $2.1(1.8-2.4)$ & $2.1(1.8-2.4)$ \\
\hline Cer linoleic (C18:2) & $0.1(0.0-0.4)$ & $0(0-0.2)$ & $0.2(0.0-0.5)$ \\
\hline Cer arachidic (C20:0) & $0.4(0.4-0.5)$ & $0.4(0.3-0.6)$ & $0.5(0.4-0.6)$ \\
\hline Cer $\alpha$-linolenic (C18:3) & $\begin{array}{c}0.18(0.16-0.22)^{\star} \\
p=0.024\end{array}$ & $0.19(0.15-0.22)$ & $0.2(0.2-0.3)$ \\
\hline Cer behenic (C22:0) & $1.2(1.1-1.4)$ & $1.4(1.2-2.2)$ & $1.3(1.1-1.4)$ \\
\hline Cer arachidonic (C20:4) & $\begin{array}{c}0.3(0.3-0.4)^{\star *} \\
p=0.006\end{array}$ & $0.3(0.1-0.5)$ & $0.4(0.3-0.5)$ \\
\hline Cer lignoceric (C24:0) & $\begin{array}{c}2.9(2.5-3.4)^{\star \star \star} \\
p=0.001\end{array}$ & $3.1(3-3.4)$ & $3.5(3.2-3.9)$ \\
\hline Cer eicosapentaenoic (C20:5) & $\begin{array}{c}0.0(0.0-0.0)^{* * *} \\
p=0.001\end{array}$ & $0.0(0.0-0.3)$ & $0.6(0-0.7)$ \\
\hline Cer nervonic (C24:1) & $2.0(1.9-2.2)$ & $2.1(2.0-3.1)$ & $2.0(1.9-2.2)$ \\
\hline Cer docosahexaenoic (C22:6) & $\begin{array}{c}0.5(0.0-0.5)^{* * *} \\
p<0.001\end{array}$ & $0.5(0.0-0.6)$ & $0.6(0.5-0.6)$ \\
\hline Total Cer & $\begin{array}{c}27.4(22.8-32.2)^{*} \\
p=0.035\end{array}$ & $27.6(23.6-35.6)$ & $32.4(26.5-34.0)$ \\
\hline Sphingosine-1-phosphate & $\begin{array}{c}508.0(438.4-556.7)^{\star} \\
p=0.017\end{array}$ & $\begin{array}{c}533.7(473.9-567.4)^{*} \\
p=0.025\end{array}$ & $445.5(424.7-473.8)$ \\
\hline
\end{tabular}


In our study we did not show statistically significant differences between the overall concentrations of serum FA in psoriatic patients with different levels of ALT. After the division of FA according to their biological properties, we found highly abnormal profiles of FA in both psoriatic groups in comparison to the healthy subjects. We also observed a positive correlation of saturated FA with serum ALT level in our examined group.

In psoriasis SFA plays a key role in exacerbation of skin lesions. Herbert et al. demonstrated in their study a crucial role of dietary delivered saturated fatty acids as an obesity-associated parameter which intensifies the severity of the disease. They proved that increasing the SFA concentration in healthy mice induces exacerbation of psoriasiform inflammation. According to the authors, SFA increased the inflammatory response by sensitizing myeloid cells in answer to proinflammatory stimuli, which in turn can cause activation of keratinocytes. They suggested that reduction of SFA in the diet diminished the psoriatic phenotype in obese mice [22].

Increased serum FFA levels are accompanied by insulin resistance leading to metabolic disorders such as obesity, dyslipidaemia, and cardiovascular diseases [23, 24]. According to the Feng et al., FFAs are the major risk factors in the development of NAFLD and its complications. The authors proved, in the group of 240 patients with NAFLD, significantly higher profiles of 12 subtypes of FFA and total FFA than in healthy controls. What is more, these patients with coexisting obesity presented significantly higher SFA (14:0, 16:0), monounsaturated FA (16:1, 18:1), and total FFA profiles than healthy controls [25]. Moreover, the authors point out that myristic acid (14:0), which belongs to the group of SFA and palmitoleic acid (16:1), is a promising indicator of liver dysfunction. In our study, palmitoleic acid was significantly higher in both psoriatic subgroups than in the controls, which might be a sensitive predictive factor for liver disease in psoriasis. Also, the higher serum SFA in psoriatic patients confirmed in our study may predispose patients to the development of liver disease. Another study showed an adverse effect of an SFA-rich diet on the liver. According to the authors, patients with nonalcoholic steatohepatitis consumed more SFA that patients with simple steatosis or healthy subjects. Furthermore, increasing dietary SFA intake in isocaloric substitution of carbohydrates or PUFA increases hepatic and visceral fat accumulation in healthy individuals [26]. Taking into account the above dependents, we may speculate that elevated serum concentration of SFA is associated with worse course of psoriasis and could be a prerogative feature of liver damage in this group of patients.

In our examined group we also demonstrated the negative correlation between UFA and ALT level. From the literature, we know that n-6 PUFA, marine-derived n-3 PUFA, and MUFA are believed to be protective factors for the risk of development of MetS [27, 28]. Kristensen et al. in their study showed a decrease in Disease Activity Score (DAS28-CRP) and PASI score after 24-week supplementation of n-3 PUFA. They also demonstrated a significant reduction of intake of non-steroidal anti-inflammatory drugs and paracetamol among patients with psoriatic arthritis compared with healthy controls [29]. Supplementation of n-3 PUFA also reduces pro-inflammatory cytokine levels (such as TNF- $\alpha$ and IL-6), improves glycaemia, and has hypolipidaemic effects [30]. Moreover, the Mediterranean diet, rich in monounsaturated fatty acids, reduces liver steatosis and improves insulin sensitivity in an insulin-resistant population with NAFLD [30, 31]. Therefore, the lower serum UFA concentration in our group of psoriatic patients on the one hand may exacerbate skin lesions and on the other hand may also lead to liver dysfunction and other systemic metabolic disturbances. Generally, we observed many FA profile disturbances (Table 3) in comparison to the healthy control group, which create a pro-inflammatory environment and may predispose psoriatic patients to NAFLD.

For the first time we have shown a positive correlation between the SFA/UFA ratio and the ALT level in psoriatic patients. In our previous study we demonstrated a positive correlation between the SFA/UFA ratio and the duration of the disease. What is more, we also observed a higher SFA/UFA ratio in psoriatic patients with obesity and hypertension [19]. The above findings together may indicate the increasing risk of liver dysfunction development and metabolic disorders with the duration of psoriasis.

In our study the total serum ceramide concentration did not correlate with the ALT level in psoriatic patients. However, we demonstrated a positive correlation between lignoceric ceramide (C24:0) and the serum ALT level. The serum concentration of myristic ceramide (C14:0) was higher in patients with high serum ALT than in healthy controls.

According to recent studies, both C14:0 and C24:0 ceramides can be involved in the pathogenesis of liver disease. Maldonado and Hernandes, in their study concerning the association of Cer with hepatic steatosis, showed that level of all ceramides correlated directly with ALT concentration. The authors highlight that the strongest correlation was observed with the myristic ceramide (C14:0). The authors even suggest that $\mathrm{C} 14: 0$ could be a novel biomarker of hepatosteatosis independently of obesity [32]. In this context, the high concentration of C14:0 observed in our psoriatic patients may be related to the liver dysfunction, and probably it could serve as a prognostic indicator of liver disease. Further studies on larger groups of patients are needed to confirm this dependence. In this retrospective study it is impossible to decide which of the findings is the primary: the increase of ALT or C14:0.

There are many potential links between Cer profile and metabolic disturbances. Some serum Cer species are associated with insulin resistance development - the major risk factor for liver steatosis. Haus et al., in their study on 
obese, diabetic patients, found a negative correlation of insulin sensitivity with total Cer concentration and with C18:0, C20:0, C24:1, and C24:0 [33]. Gastric bypass resulted in reduced plasma levels of C14:0, C16:0, C20:0, and C24:0 in patients. Moreover the decrease in C24:0 correlated with the amount of weight loss and the improvement in insulin sensitivity [34]. Hanamatsu et al., in their study on young obese adults, observed a positive correlation between C24:0 and $\gamma$-glutamyl transpeptidase [35]. Brozinick et al. demonstrated that the total Cer level and the levels of C14:0, C16:0, C22:0, and C24:0 were high in prediabetic and diabetic nonhuman primates, and that the levels of these Cer correlate with the homeostasis model assessment of insulin resistance (HOMA-R) [36]. All these findings suggest that C24:0 and C14:0 may have a great impact on creating metabolic disturbances, also among psoriatic patients.

We observed higher serum concentration of S1P in psoriatic patients with high serum ALT than in healthy controls. S1P is a bioactive signalling molecule, and it plays a role as a key mediator of many physiological and pathophysiological responses, such as cell growth and survival, differentiation, migration, vascular integrity, lymphocyte trafficking, and immune response. The role of S1P is to inhibit keratinocyte proliferation and induce differentiation and migration of the above cells. Its higher level was described in many metabolic disturbances and was indicated as a marker of obesity and insulin resistance $[37,38]$. Recently it was also found to play an important role in liver pathology, such as acute liver failure, liver fibrosis, and fatty liver disease [39]. Our results indicate that S1P may be involved in the development of liver disease also in psoriatic patients.

There are some limitations of our study. Due to the fact that this is a retrospective study, in the hospital documentation we had only alanine transaminase serum concentration. We did not perform ultrasonography or biopsy of the liver. Taking into account the fact that we excluded patients with other potential, secondary causes of liver damage, it can be considered with high probability that the above disturbances occurred in the course of psoriasis. Our results need confirmation in further studies.

\section{Conclusions}

Patients with severe psoriasis are predisposed to the development of liver dysfunction. We demonstrated disturbances of serum fatty acids and sphingolipid profile in psoriatic patients, which may trigger liver disease. An abnormal FA profile might provide some additional predictive value for liver dysfunction and may convey higher risk of NAFLD development in this group of patients.

\section{Acknowledgments}

This study was supported by a study grant from the Medical University of Białystok (Project No: N/ST/ ZB/18/001/1149).

\section{Conflict of interest}

The authors declare no conflict of interest.

\section{References}

1. Sales R, Torres T. Psoriasis and metabolic syndrome. Acta Dermatovenerol Croat 2014; 22: 169-74.

2. Gerdes S, Mrowietz U, Boehncke WH. Comorbidity in psoriasis. Hautarzt 2016; 67: 438-44.

3. Fernández-Armenteros JM, Gómez-Arbonés X, Buti-Soler M, et al. Psoriasis, metabolic syndrome and cardiovascular risk factors. A population-based study. J Eur Acad Dermatol Venereol 2019; 33: 128-35.

4. Correia B, Torres T. Obesity: a key component of psoriasis. Acta Biomed 2015; 86: 121-9.

5. Boehncke WH. Systemic inflammation and cardiovascular comorbidity in psoriasis patients: causes and consequences. Front Immunol 2018; 9: 579.

6. Yki-Järvinen H. Non-alcoholic fatty liver disease as a cause and a consequence of metabolic syndrome. Lancet Diabetes Endocrinol 2014; 2: 901-10.

7. Dietrich P, Hellerbrand C. Non-alcoholic fatty liver disease, obesity and the metabolic syndrome. Best Pract Res Clin Gastroenterol 2014; 28: 637-53.

8. Boppidi H, Daram SR. Nonalcoholic fatty liver disease: hepatic manifestation of obesity and the metabolic syndrome. Postgrad Med 2008; 120: E01-7.

9. Ganzetti G, Campanati A, Offidani A. Non-alcoholic fatty liver disease and psoriasis: so far, so near. World J Hepatol 2015; 7: 315-26.

10. Ganzetti G, Campanati A, Molinelli E, et al. Psoriasis, nonalcoholic fatty liver disease, and cardiovascular disease: three different diseases on a unique background. World J Cardiol 2016; 8: 120-31

11. Mantovani A, Gisondi P, Lonardo A, et al. Relationship between non-alcoholic fatty liver disease and psoriasis: a novel hepato-dermal axis? Int J Mol Sci 2016; 17: 217.

12. Gisondi P, Targher G, Zoppini G, et al. Non-alcoholic fatty liver disease in patients with chronic plaque psoriasis. Hepatol 2009; 51: 758-64.

13. Madanagobalane S, Anandan S. The increased prevalence of non-alcoholic fatty liver disease in psoriatic patients: a study from South India. Australas I Dermatol 2012; 53: 190-7.

14. Abedini R, Salehi M, Lajevardi V, et al. Patients with psoriasis are at a higher risk of developing nonalcoholic fatty liver disease. Clin Exp Dermatol 2015; 40: 722-7.

15. Candia R, Ruiz A, Torres-Robles R, et al. Risk of non-alcoholic fatty liver disease in patients with psoriasis: a systematic review and meta-analysis. J Eur Acad Dermatol Venereol 2015; 29: 656-62.

16. Berlanga A, Guiu-Jurado E, Porras JA, et al. Molecular pathways in non-alcoholic fatty liver disease. Clin Exp Gastroenterol 2014; 7: 221-39.

17. Pagadala MT, Kasumov T, McCullough AJ, et al. Role of ceramides in nonalcoholic fatty liver disease. Trends Endocrinol Metab 2012; 23: 365-71.

18. Marí M, Fernández-Checa JC. Sphingolipid signalling and liver diseases. Liver Int 2007; 27: 440-50.

19. Myśliwiec H, Baran A, Harasim-Symbor E, et al. Serum fatty acid profile in psoriasis and its comorbidity. Arch Dermatol Res 2017; 309: 371-80.

20. Myśliwiec H, Baran A, Harasim-Symbor E, et al. Increase in circulating sphingosine-1-phosphate and decrease in ce- 
ramide levels in psoriatic patients. Arch Dermatol Res 2017. 309: 79-86.

21. Glaser C, Demmelmair H, Koletzko B. High-throughput analysis of total plasma fatty acid composition with direct in situ transesterification. PLoS One 2010; 5: e12045.

22. Herbert D, Franz S, Popkova Y, et al. High fat diet exacerbates early psoriatic skin inflammation independent of obesity: saturated fatty acids as key players. J Invest Dermatol 2018; 138: 1999-2009.

23. Boden G. Obesity and free fatty acids. Endocrinol Metab Clin North Am 2008; 37: 635-46.

24. Boden G. Obesity, insulin resistance and free fatty acids. Curr Opin Endocrinol Diabetes Obes 2011; 18: 139-43.

25. Feng R, Luo C, Li C, et al. Free fatty acids profile among lean, overweight and obese non-alcoholic fatty liver disease patients: a case-control study. Lipids Health Dis 2017; 16: 165.

26. Della Pepa G, Vetrani C, Lombardi G, et al. Isocaloric dietary changes and non-alcoholic fatty liver disease in high cardiometabolic risk individuals. Nutrients 2017; 9: 1065.

27. Kuo-Liong C, Chia-Lun C, Chen-Hong K, et al. Plasma fatty acids and the risk of metabolic syndrome in ethnic Chinese adults in Taiwan. Lipids Health Dis 2011; 10: 33.

28. Baran A, Kiluk P, Myśliwiec H, et al. The role of lipids in psoriasis. Dermatol Rev 2017; 104: 619-35.

29. Kristensen S, Schmidt EB, Schlemmer A, et al. Beneficial effect of $n-3$ polyunsaturated fatty acids on inflammation and analgesic use in psoriatic arthritis: a randomized, double blind, placebo-controlled trial. Scand I Rheumatol 2018; 47: 27-36.

30. O'Mahoney LL, Matu J, Price OJ, et al. Omega-3 polyunsaturated fatty acids favourably modulate cardiometabolic biomarkers in type 2 diabetes: a meta-analysis and metaregression of randomized controlled trials. Cardiovasc Diabetol 2018; 17: 98.

31. Ryan MC, Itsiopoulos C, Thodis T, et al. The Mediterranean diet improves hepatic steatosis and insulin sensitivity in individuals with non-alcoholic fatty liver disease. J Hepatol 2013; 59: 138-43.

32. Maldonado-Hernández J, Saldańa-Dávila GE, Pińa-Aguero MI, et al. Association between plasmatic ceramides profile and AST/ALT ratio: C14:0 ceramide as predictor of hepatic steatosis in adolescents independently of obesity. Can J Gastroenterol Hepatol 2017; 2017: 3689375.

33. Haus JM, Kashyap SR, Kasumov T, et al. Plasma ceramides are elevated in obese subjects with type 2 diabetes and correlate with the severity of insulin resistance. Diabetes 2009; 58: 337-43.

34. Huang H, Kasumov T, Gatmaitan P, et al. Gastric bypass surgery reduces plasma ceramide subspecies and improves insulin sensitivity in severely obese patients. Obesity 2011; 19: $2235-40$

35. Hanamatsu H, Ohnishi S, Sakai S, et al. Altered levels of serum sphingomyelin and ceramide containing distinct acyl chains in young obese adults. Nutr Diabetes 2014; 4: e141.

36. Brozinick JT, Hawkins E, Hoang Bui H, et al. Plasma sphingolipids are biomarkers of metabolic syndrome in non-human primates maintained on a Western-style diet. Int J Obes 2013; 37: 1064-70.

37. Majumdar I, Mastrandera LD. Serum sphingolipids and inflammatory mediators in adolescents at risk for metabolic syndrome. Endocrine 2012; 41: 442-9.

38. Kowalski G, Carey AL, Selathurai A. Plasma sphingosine1-phosphate is elevated in obesity. PLoS One 2013; 8 : e72449.
39. Rohrbach T, Maceyka M, Spiegel S. Sphingosine kinase and sphingosine-1-phosphate in liver pathobiology. Crit Rev Biochem Mol Biol 2017; 52: 543-53. 